Linguistique, littérature, didactique

$157-158 \mid 2013$

Théories et pratiques des genres

\title{
Genre, généricité et compétence lectoriale
}

\section{Estelle Riquois}

\section{(2) OpenEdition}

\section{Journals}

Édition électronique

URL : http://journals.openedition.org/pratiques/3832

DOI : $10.4000 /$ pratiques.3832

ISSN : 2425-2042

\section{Éditeur}

Centre de recherche sur les médiations (CREM)

\section{Édition imprimée}

Date de publication : 1 juin 2013

Pagination : 177-188

\section{Référence électronique}

Estelle Riquois, « Genre, généricité et compétence lectoriale », Pratiques [En ligne], 157-158 | 2013, mis en ligne le 18 décembre 2017, consulté le 19 avril 2019. URL : http://journals.openedition.org/ pratiques/3832 ; DOI : 10.4000/pratiques.3832 


\title{
Genre, généricité et compétence lectoriale
}

\section{Estelle Riquois}

\author{
Université Paris Descartes, laboratoire EDA
}

Pour de nombreux enseignants de français langue étrangère, l'exploitation pédagogique du texte littéraire en classe a longtemps été synonyme de retour en arrière, de cours traditionnel et d'apprenant peu motivé. Vue comme un territoire hostile trop difficile d'accès, elle ne séduisait pas des enseignants tournés vers l'oral qui l'avaient exclu de leurs classes. Dans les années 1970, l'approche communicative a pourtant courageusement réintroduit les textes littéraires, désormais considérés comme des documents authentiques qui permettent aux apprenants d'accéder directement à des textes destinés à l'origine à des locuteurs natifs.

Utilisé dans les classes au même titre que le texte de presse ou la publicité, le texte littéraire ne se lit toutefois pas selon les mêmes modalités et s'accompagne de notions spécifiques et d'une histoire qui peuvent rendre son exploitation pédagogique complexe pour l'enseignant.

Pourtant, pour l'enseignement-apprentissage du FLE, la littérature a bien des avantages. Dans de nombreuses situations d'apprentissage, la pratique de la langue cible en dehors de la classe se fait essentiellement à l'écrit, car il peut être difficile de rencontrer des locuteurs francophones, l'accès à Internet n'est pas toujours assuré financièrement ou techniquement, tandis que de nombreux établissements disposent de bibliothèques où des ouvrages variés sont à disposition. Il n'est toutefois pas toujours simple pour un apprenant de langue étrangère de lire un texte long. Les stratégies de lecture dont il dispose en langue maternelle ne sont pas immédiatement transférables lorsqu'il lit un texte en langue cible et un bon lecteur peut éprouver des difficultés qui lui sont inconnues en langue maternelle. La lecture d'un texte littéraire dépasse également la simple compréhension du texte, nécessitant des savoir-faire qu'il faut se réapproprier en langue étrangère pour disposer des outils nécessaires à la lecture de ces ouvrages, développant ainsi une compétence permettant de poursuivre son apprentis- 
sage hors de la classe. Territoire de réflexion, d'interrogation, d'investissement de soi ${ }^{(1)}$, la littérature offre un espace où la lecture peut se développer de manière authentique, où l'apprenant peut rencontrer le texte comme peut le faire un natif.

Mais alors vient la question essentielle pour l'enseignant : comment enseigner pour amener l'apprenant à développer cette compétence ? La compétence de lecture ne suffit pas pour disposer des outils nécessaires à l'apprenant pour devenir un vrai lecteur. Savoir lire permet de déchiffrer le texte, il faut ensuite le comprendre et l'interpréter, et pourquoi pas, avant même la lecture, pouvoir l'identifier et le choisir. Comme le montre les études sociologiques sur la question ${ }^{(2)}$, pour un locuteur natif savoir lire ne donne pas accès à tous les lieux de mise à disposition des livres. Une librairie ou une bibliothèque sont des espaces codés où les livres sont plus aisément accessibles à ceux qui savent les reconnaitre. Le paratexte, le classement des ouvrages peut devenir un labyrinthe pour le lecteur volontaire mais peu coutumier du lieu, et l'on comprend que de nombreux apprenants ne poussent pas la porte de la bibliothèque. Plongés dans un univers éditorial qui ne leur est pas familier, adoptant un statut hybride d'apprenant et de lecteur, ils ne peuvent s' appuyer sur leurs connaissances et les transposer simplement dans une situation différente.

Partant de ce constat, il paraît intéressant d'enseigner une compétence plus large, qui dépasse la lecture entendue comme la compréhension du texte, en donnant accès à des outils utiles tout autant avant que pendant cette lecture. Cette conception étendue de la compétence de lecture, nommée « compétence lectoriale » (Riquois, 2010a) en reprenant la terminologie utilisée par J.-M. Schaeffer à propos de l'aspect générique de la lecture, est une compétence où le genre joue un rôle important. À différents niveaux sur lesquels nous allons revenir, le genre constitue un champ de connaissance nécessaire à l'apprenant pour qu'il devienne lecteur à part entière, pour qu'il s'engage dans sa lecture en langue étrangère comme il peut le faire en langue maternelle. Il invite aussi à s'interroger sur l'appartenance générique des textes, car elle conditionne en partie leur réception. C'est pourquoi nous aborderons dans un premier temps la notion de genre et de généricité, puis nous placerons cette notion dans le champ de la didactique, avant de prendre pour exemple le roman policier.

\section{Genre et généricité}

L'appartenance d'un texte à un genre peut revêtir une grande importance, et celle-ci va dépendre du point de vue adopté.

Pour l'auteur, inscrire son texte dans un genre peut lui permettre de signifier

(1) A. Séoud engage l'enseignant à se rapprocher le plus possible de la situation de réception authentique du document en développant «l'investissement de soi, la recherche de l'émotion, l'engagement de la sensibilité [car] en littérature on lit rien que par amour de la lecture, et seulement pour son propre plaisir» (1997:66). Ce n'est pas toujours possible, mais ce point de vue a le mérite de remettre le plaisir de lire au premier plan.

(2) Cf. notamment l'étude de J.-F. Hersent (2000) La sociologie de la lecture en France, qui nous apprend que $29 \%$ de français fréquentent les librairies indépendantes, tandis que $24 \%$ achètent leurs livres en grande surface alimentaire. 
son appartenance à la littérature conçue comme un espace élitiste opposé à la paralittérature, ou au contraire lui donner l'occasion de revendiquer une autre position. Comme l'écrit J.-M. Schaeffer « la véritable raison de l'importance accordée par la critique littéraire à la question du statut des classification [...] réside dans le fait que [...] la question de savoir ce qu'est un genre littéraire (et du même coup celle de savoir quels sont les "véritables" genres littéraires et leurs relations) est censée être identique à la question de savoir ce qu'est la littérature » (1989: 8). L'écrivain a donc un choix à faire qui peut lui permettre d' exprimer une revendication littéraire, contredire ses textes précédents, ou aller à l'encontre de l'affichage choisi par l'éditeur.

Pour le lecteur, l'identification du genre va fournir un faisceau d'informations plurielles qui vont agir sur la perception du livre (et parfois aussi de l'auteur) avant la lecture, sur la réception ensuite et sur la perception, voire l'interprétation du texte lu. La couverture annonce, le texte confirme ou contredit, le lecteur reçoit et interprète. Ces différents temps sont interdépendants et le positionnement d'une œuvre se fait au sein d'une hiérarchie littéraire reposant sur la valeur supposée d'un texte. Depuis le XIX siècle, le clivage que décrit P. Bourdieu dans Les règles de l'art (1992) a fortement marqué la réception des œuvres littéraires. À cette époque, un espace autonome restreint au fort capital symbolique s'est constitué au cœur du champ de production culturelle, formé par la production élitiste reconnue par l'institution littéraire. Le reste de la production, jugé de moins bonne qualité, a été rejeté de cet espace, constituant un faire-valoir ou un repoussoir pour la production élitiste. Cette conception perdure aujourd'hui en France, blâmant le succès de librairie pour encenser l'ouvrage élitiste au public plus restreint.

Dans cette conception où « la bonne littérature, c'est la littérature tout court » (Ansel, $2005: 74$ ), l'hétérogénéité effective de la production n'est pas prise en compte. La remise en question des marges, notamment, est gommée alors que de plus en plus d'écrivains les interrogent en proposant des textes hybrides, affichant une appartenance générique que le texte lui-même peut contredire.

Mais n'est-ce pas directement l'institution littéraire qui est remise en question par ces tentatives de franchissement de frontière? Le texte reconnu comme étant littéraire représente l'institution et s'inscrit sur une échelle de valeur dont elle est garante. Le texte et son auteur sont partie prenante de ce système, où littérature et paralittérature constituent deux premiers niveaux d'identification, affinés ensuite par la distinction des différents genres littéraires eux-mêmes hiérarchisés ${ }^{(3)}$.

Cette échelle de valeur n'est cependant valable que pour un temps donné qui semble actuellement toucher à sa fin, car selon B. Tomachevski « le remplacement constant des genres élevés par des genres vulgaires appartient au processus de succession des genres » (1965:309). L'auteur indique également que ce processus peut prendre la forme d'une incursion du genre vulgaire dans le genre élevé. Il appartient alors au lecteur de connaitre aussi le genre en question pour pou-

(3) Au sein de la littérature, les genres classiques placent la poésie au sommet de la hiérarchie, tandis que le roman policier occupe la position la plus valorisante parmi les genres paralittéraires, suivi de près par le roman de science-fiction. 
voir identifier cette évolution, mais il est intéressant d'observer que la notion de genre ne lui permettra pas de décrire ce phénomène. Dire par exemple d'un roman qu'il appartient au genre romanesque ne donne aucune information à son lecteur sur les emprunts éventuels que l'auteur aura pu faire au roman policier. Cela aura pourtant une grande importance pour la réception du texte car c'est ainsi que le lecteur peut construire un horizon d'attente qui va interagir avec le texte.

Pour analyser ce phénomène, J.-M. Schaeffer (1989: 151) propose d'opposer le « régime auctorial et le régime lectorial» de généricité. Ils permettent de s'intéresser d'une part aux choix de l'auteur, et d'autre part aux choix du lecteur. Il ne s'agit pas uniquement de rendre compte d'une classification, mais de montrer que l'inscription générique d'un texte a une portée plus importante. Ainsi, du côté de l'auteur, il s'agit de décrire et d'analyser les choix génériques affichés dans le texte, les relations que l'auteur a décidé d'établir entre son écriture et un ou plusieurs genres. Il peut s'agir d'une inscription générique conforme au modèle ou au contraire d'une transgression revendiquée. Du côté du lecteur, le régime lectorial de généricité est entendu comme une interprétation du texte liée à l'horizon d'attente générique de celui-ci plutôt qu'à une simple classification. Le lecteur identifie les signes qui relèvent de la connaissance générique partagée avec l'auteur, et leur donne un sens qui n'est pas nécessairement celui qui était attendu.

Pour aller plus loin et tenir compte d'un troisième acteur intervenant dans ce processus, J.-M. Adam et U. Heidmann (2007) complètent ce modèle par un « régime de généricité éditoriale » qui va jouer un rôle dans l'existence de l'œuvre, dans son appartenance générique effective et dans son interprétation. Ce troisième régime est entendu comme «l'action médiatrice capitale de la diffusion par le moyen d'un médium écrit, numérisé ou audio-visuel » (24). La collection, la couverture, l'objet-livre sont autant d'éléments qui comptent au moment de la lecture et qui peuvent parfois être très signifiants. Confié à un éditeur, le texte subit des transformations et devient un produit qui doit attirer un public. Il peut évoluer et connaitre des modifications importantes de format, de collection, voire de titre. Le texte originel ne change quasiment pas, mais l'évolution de sa présentation peut entrainer une évolution de sa réception par les lecteurs. Il est également intéressant de remarquer que le rôle de l'éditeur est fréquemment oublié par les lecteurs. Il n'est pas considéré comme un acteur agissant dans la chaine du livre, bien qu'il remplisse un rôle essentiel dans la mise à disposition du texte.

Si l'on tient compte de ces propositions, la notion de genre apparait trop restrictive. Elle indique une appartenance fermée qui ne laisse pas de place aux phénomènes que nous venons de décrire. Pourtant, son utilisation est utile pour le lecteur, comme pour l'apprenant que nous allons évoquer ensuite.

Toute transgression, tout écart par rapport à une norme, nécessite effectivement de pouvoir se référer à un modèle pour mesurer cet écart. Le modèle ne peut donc pas être supprimé, sous peine de ne plus disposer de repère pour l'analyse. En revanche, il est possible d'interroger les catégories qu'impose une structure hiérarchisée comme celle-ci, afin de déterminer son fonctionnement et les implications de son utilisation. 
Reste alors à définir ces catégories génériques. A. Compagnon dans son cours sur le genre indique que " depuis les environs du XIX ${ }^{\mathrm{e}}$ siècle, [le système moderne des genres] se réduit en effet et de plus en plus à trois grandes cases fourretout : la poésie, le récit et le théâtre » [2007 : en ligne]. Ces trois catégories assurément «fourre-tout» semblent dès lors pouvoir être perçues comme de simples étiquettes servant à classer grossièrement les œuvres publiées. Pourtant, nous l'avons dit plus haut, ce sont des outils efficaces pour l'interprétation des textes, tout en étant également prescripteurs. Pour trouver son lectorat, il est important que l'auteur inscrive son texte dans un genre reconnu par l'institution, y compris s'il le transgresse ensuite. Dans le cas contraire, il prend le risque de ne pas être lu par manque de visibilité, ou de ne pas être compris par les lecteurs.

Il semble en résulter une permanence du modèle apparemment contradictoire avec ces transformations constantes observées dans les œuvres. Le genre resterait immuable, mais ses manifestations évolueraient. Or, il n'en est rien. Si les étiquettes restent, ce qu'elles recouvrent pour leurs utilisateurs prend de nouvelles formes. L'art poétique tel que l'entendait Aristote ne correspond plus à la poésie actuelle. L'espace désigné par le genre est en perpétuelle mutation, mais l'auteur inclue toujours un signe qui permet de placer son texte dans une relation au genre auquel il entend identifier sa production. La généricité ne peut par conséquent fonctionner que dans une relation au genre conçu comme une catégorie symbole de l'institution littéraire.

Cela implique également que le paysage littéraire ne puisse plus être vu comme un espace double constitué de deux groupes hermétiques de textes, la littérature s'opposant à la paralittérature, dans une relation dévalorisante pour la seconde. Comme le dit D. Combe : «c'est peut-être aujourd'hui le propre des œuvres littéraires importantes, ambitieuses, que d'être mixtes par nature » (1992: 151). Ces œuvres mixtes interdisent toute catégorisation trop stricte tout en conservant un lien fort avec le genre transgressé, et invitent à voir la littérature comme un espace unique où s'exprime une généricité foisonnante. Les genres traditionnels deviennent alors des outils d'analyse et d'interprétation qui n'enferment pas tout en permettant l'identification.

Le régime de généricité permet de disposer d'un outil d'analyse qui enrichit la représentation de la notion de genre. L'auteur, l'éditeur et le lecteur sont considérés comme des instances agissantes qui participent tous trois à la production et à la réception du texte. Dans cette perspective, tout texte est susceptible de comporter des éléments pouvant être rattachés à plusieurs genres et l'objet-livre peut être interrogé au même titre que le texte.

Il appartient au lecteur de pouvoir identifier le ou les genres convoqués par l'auteur avant et pendant sa lecture, s'il veut disposer de l'ensemble des informations nécessaires à la réception du texte. Ce faisant, il pourra s'approcher du lecteur modèle envisagé lors de l'écriture (Eco, 1985) et tendre vers une lecture proche de ce qu'envisageait l'auteur.

Cela n'est évidemment pas indispensable, mais comme nous allons le voir, l'apprenant de français langue étrangère pourra bénéficier d'un enseignement qui s'appuie sur les genres et la généricité pour devenir lui aussi lecteur à part entière en langue cible, et enrichir sa compétence lectoriale en langue maternelle. 


\section{Généricité et lecture littéraire}

Pour l'enseignant, la question posée lors de l'introduction de cet article reste encore entière à ce stade, car reconnaitre l'importance du genre et de la généricité dans le processus de lecture n'indique pas comment enseigner une compétence «générique » en langue étrangère qui permettrait aux apprenants d'être des lecteurs à part entière. Face à un texte écrit, l'objectif immédiat fixé en classe de langue étrangère porte généralement sur le décodage et la compréhension. À partir de cette étape, d'autres activités sont envisagées par l'enseignant pour permettre à l'apprenant d'enrichir ses compétences en langue. Lorsque le support pédagogique est un texte littéraire, présenté en version intégrale ou sous la forme d'un extrait, on observe fréquemment dans les manuels une exploitation pédagogique identique ${ }^{(4)}$, qui s'éloigne de la lecture authentique de ce type de texte, et des préconisations de la perspective actionnelle qui se développe actuellement dans les classes de langue étrangère.

Pourtant, pour un public adolescent et adulte en classe de FLE non spécifique, nous l'avons dit, l'accès à la production littéraire française et francophone peut représenter un atout de taille pour la poursuite autonome de l'apprentissage de la langue cible et pour son approfondissement. S'appuyant sur les pratiques des apprenants et sur le transfert de stratégies disponibles en langue maternelle, l'apprentissage d'une compétence lectoriale vise à exploiter la lecture littéraire pour devenir réellement acteur de sa lecture, comme le fait un lecteur natif.

Or, lorsqu'il est question de littérature, le lecteur adopte un mode de lecture particulier. Le texte littéraire n'est pas lu pour s'informer, comme peut l'être un journal, ou pour chercher une information technique que l'on trouve dans des notices, par exemple. L'auteur impose au lecteur un pacte de lecture qui l'engage à accepter l'illusion référentielle, l'identification éventuelle aux personnages et une adhésion au texte. Dans le cadre d'une classe, l'apprenant, lecteur autonome à l'extérieur de la classe, se voit soumis au choix de l'enseignant et à la situation d'apprentissage qui modifie sa relation au texte. Le texte est alors lu parce que l'enseignant le demande, et non parce que l'apprenant l'a choisi. L'objectif associé à la lecture se déplace également pour en faire uniquement un support d'apprentissage. Dans ces conditions, cette lecture d'apprentissage ou « scolaire» semble très éloignée d'une lecture «plaisir » qui relèverait plutôt de l'espace privé et de l'acte individuel.

Cette « lecture plaisir», souvent citée comme un idéal, ne correspond toutefois pas davantage à la lecture littéraire. Un lecteur peut trouver plaisante la lecture d'un magazine, sans avoir recours aux stratégies de lecture propres à la réception d'un texte littéraire. Car il s'agit bien de stratégies spécifiques qui correspondent à un type de document particulier. Selon J.-L. Dufays, « la littérature réside moins dans le texte que dans sa lecture. [...] la littérature est un effet de lecture qui dépend de l'attitude qu'on adopte à l'égard du texte. Aucun texte n’est littéraire en soi » (1994:213). Se plaçant ici du côté de la situation de ré-

(4) L'analyse d'un corpus de 52 manuels de français langue étrangère édités en France entre 1999 et 2008 a permis de dégager 1'exploitation pédagogique assignée à chaque genre pour les niveaux 1 à 4 (cf. Riquois, 2009). 
ception, l'auteur affirme ainsi que le caractère littéraire du texte dépend des stratégies de lecture employées par le lecteur, de son attitude par rapport au texte. Nous l'avons dit plus haut, cette attitude est également influencée par l'identification du texte avant même la lecture. Le texte se présente accompagné d'un paratexte qui permet d'anticiper la lecture en assignant au texte certaines caractéristiques et invite le lecteur à adopter une attitude spécifique adaptée à ce type de texte.

C'est ici que le genre et la généricité apparaissent comme des outils efficients pour les apprenants. Les stratégies de lecture utilisées en langue maternelle ne sont pas toujours transférées telles quelles en langue étrangère. Si la compréhension du texte est atteinte en fonction des compétences linguistiques de l'apprenant, l'interprétation, voire l'appropriation du texte, nécessitent des stratégies spécifiques pour être effectivement réalisées. Or, quelque soit le degré de maîtrise de ces stratégies en langue maternelle et en dehors de la classe, pour de nombreux apprenants, il sera nécessaire de procéder d'abord à une phase d'explicitation des manières de faire pour passer de la phase de compréhension liée à l'apprentissage, à une phase d'interprétation des informations paratextuelles et textuelles. Le contexte d'apprentissage impose effectivement une attitude particulière face au texte, où l'apprenant s'attend à ce qu'on lui pose des questions, ou envisage la lecture comme un préalable pour une autre activité. Cette attitude permet de placer l'apprenant dans une posture de questionnement, interrogeant le contenu du texte en convoquant certaines stratégies de lecture habituellement inconscientes.

La connaissance de ces stratégies donne des outils à l'enseignant pour accompagner ses apprenants vers l'accès au texte et à une lecture autonome. En enseignant ces stratégies, et en convoquant des stratégies déjà utilisées en langue maternelle, il permet la constitution d'un répertoire de compétences, au sein duquel la compétence générique va avoir toute sa place.

Identifier l'appartenance générique d'un texte se révèle effectivement indispensable avant et pendant la lecture quelque soit la langue du texte. Face à celuici, le lecteur fait des hypothèses, anticipe en envisageant un domaine de référence et en adoptant une posture en adéquation avec le document. Les textes littéraires nécessitent ce travail de la part de leur lecteur. Par le paratexte, le choix du titre, le nom de l'auteur, celui de l'éditeur, ils s'adressent à un lectorat censé leur correspondre et orientent ses attentes en utilisant des normes typologiques et des invariants génériques. Ces informations peuvent être contredites ensuite, mais elles doivent donner envie au lecteur de poursuivre sa découverte du texte.

Pendant la lecture en classe, les connaissances de l'apprenant concernant les genres sont également convoquées et donnent des outils pour observer la conformité au genre affiché, ou au contraire, pour mesurer les écarts éventuels, les généricités qui se mêlent ou le contournement des normes. L'apprenant prend alors une posture de lecteur, puisqu'il peut répondre aux sollicitations de l'auteur. Il n'est plus seulement question de lire et de comprendre, mais d'interpréter, d'analyser, sans toutefois perdre le plaisir de lire.

Cette posture spécifique au texte littéraire donne accès à un mode de lecture courant en dehors de la classe, et souhaitable également dans le cadre de l'apprentissage. Il n'est plus question de sacraliser l'écrit littéraire mais d'enseigner 
les outils permettant de restituer des situations de lecture aussi proches que possible de l'authenticité de la réception de la littérature. Dans cette perspective, l'apprenant doit parvenir à maitriser un mode d'accès au texte qui conçoit la lecture comme un "va-et-vient dialectique » entre deux modes de lecture, entre l'interprétation et l'identification, entre l'analyse et la lecture « plaisir ».

Selon Dufays, Gemenne et Ledur (2005 : 93), la lecture en classe ne permet pas à priori de mettre en place cette relation au texte, comme nous l'avons observé dans de nombreux manuels de FLE (Riquois, 2009). Le cadre de la classe implique un travail «sur » le texte, une distanciation qui doit permettre l'analyse. En conséquence, les activités proposées dans les manuels relèvent davantage de la compréhension puis de la manipulation linguistique, que de l'interprétation du texte, reléguant la lecture-participation à l'extérieur de la classe, l'illusion référentielle et le plaisir de lire ne semblant pas y avoir de place.

Or, pour que la lecture soit réellement littéraire, pour qu'elle réponde au jeu instauré par l'auteur, il est préférable qu'elle soit envisagée comme un équilibre entre participation et distanciation, entre plaisir de lire et interprétation, y compris en classe. C'est à cette condition que l'apprenant peut réellement remplir son rôle d'acteur dans la relation au texte et devenir lecteur. En procédant de la sorte, il actualise le texte en lui donnant une interprétation personnelle, en le faisant sien, en acceptant le contrat de lecture proposé par l'auteur. A l'inverse, aucune interprétation n'est définitive ou « juste », la parole de l'enseignant ne fait pas autorité si l'apprenant maitrise les présupposés du texte et peut répondre aux sollicitations de l'auteur dont il a repéré les questionnements.

En langue étrangère, l'apprenant n'aura pas toujours les compétences attendues ou nécessaires pour mettre en place ce mode de lecture, et la lecture-plaisir pourra sembler inutile dans le processus d'apprentissage. Pourtant, le plaisir de lire est une source importante de motivation qui peut être utilement associée à une distance critique où les apprentissages sont possibles, notamment en ce qui concerne les stratégies.

Il faut alors disposer de savoirs et de savoir-faire précis, d'outils qui permettent à l'apprenant de mettre en œuvre ses connaissances et de les compléter pour former une compétence générique valide pour sa langue maternelle comme pour les langues étrangères qu'il pratique.

La classification générique peut en effet varier d'une culture à l'autre, impliquant des connaissances spécifiques liées au contexte de réception supposé, mais les savoir-faire sont les mêmes et peuvent être consciemment transposés d'une langue à l'autre. Ce travail comparatif pourra également être bénéfique pour la lecture en langue maternelle.

Enseigner une compétence générique en classe de français langue étrangère peut donc offrir aux apprenants des outils destinés à faciliter la mise en place d'une réelle posture de lecteur. La lecture littéraire conçue comme un jeu, comme un espace ouvert d'interprétation et d'analyse devient le lieu de la rencontre avec le texte et d'une prise de position de l'apprenant. L'identification générique du texte peut ainsi intervenir avant la lecture selon les documents choisis par l'enseignant, permettant au futur lecteur de choisir ce qu'il va lire, d'anticiper cette lecture et de pouvoir ensuite confronter ses hypothèses avec la réalité du texte. 


\section{Développer une compétence générique par la lecture et l'écriture de nouvelles policières}

Pour l'enseignant qui choisit de développer des situations où la lecture littéraire pourra trouver sa place, il importe également de sélectionner des objectifs d'apprentissage pertinents dans un cours de français langue étrangère non spécifique. L'apprentissage de la langue étant l'objet principal de son enseignement, un cours formel sur l'histoire littéraire ou sur l'analyse littéraire est exclue, mais le transfert des compétences acquises en langue maternelle, notamment en ce qui concerne les genres littéraires, est souhaitable.

Dans ce cadre, pour le chercheur, il pourra être intéressant d'observer dans un premier temps ce qui se fait dans la classe ${ }^{(5)}$, pour mesurer ensuite l'impact d'un enseignement apprentissage visant le transfert de savoirs et de savoir-faire de la langue maternelle à la langue cible et le développement d'une posture de lecteur littéraire chez les apprenants par l'étude du genre et de la généricité d'un texte.

Pour cela, nous nous sommes appuyé sur une analyse préalable de séquences descriptives à partir d'un corpus de descriptions urbaines extraites de romans policiers et de romans réalistes du XIX ${ }^{\mathrm{e}}$ siècle $^{(6)}$. Cette analyse a montré la porosité des genres et a permis de mettre en valeur les caractéristiques prototypiques de certains textes et la généricité développée par quelques auteurs à partir de ces caractéristiques.

Le choix du roman policier permet ici de disposer d'une référence connue des apprenants (au besoin, l'enseignant peut avoir recours au modèle cinématographique) et d'une structure romanesque claire et déployant le schéma de l'enquête et de la prise d'indice, ce qui est également demandé en classe à l'apprenant, notamment lors d'observations grammaticales. L'apprentissage s'appuie ainsi sur un exercice fréquent pour le complexifier et lui assigner un nouvel objectif. Les enquêtes menées auprès des apprenants à la suite de ces activités ont également montré que le roman policier est considéré comme un genre «facile» qui permet d'entrer dans la lecture avant de lire des textes plus complexes comme ceux de Flaubert. Certains textes policiers contredisent cet a priori, mais grâce à cette disposition d'esprit, leur utilisation en classe était facilitée.

Ce corpus de texte a ensuite été exploité en classe auprès d'un public d'étudiants allophones résidant en France pour un semestre dont le niveau est équivalent au niveau B2 du CECRL. L'objectif principal de cette exploitation était le développement d'une compétence lectoriale, notamment en développant un savoir-faire générique. Pour cela, les activités proposées aux apprenants ont d'abord porté sur la réception, sur la lecture et l'interprétation des textes, avant de

(5) L'observation de manuels donne des informations dans ce domaine, et montre que le genre majoritaire est le récit romanesque, suivi par la poésie, mais qu'il n'est que rarement question de travailler sur le genre lui-même ( $c f$. Riquois, 2009).

(6) Cette analyse détaillée réalisée lors de notre recherche doctorale (Pour une didactique des littératures en français langue étrangère : du roman légitimé au roman policier, Éditions Universitaires Européennes, 2010) est présentée dans 1'article « La linguistique textuelle au service des genres littéraires ", dans les actes du colloque Linguistique et littérature : Cluny, 40 ans après (2010b). 
s'orienter vers la production et l'écriture créative destinée à s'approprier pleinement les savoir-faire acquis pendant le projet.

Le corpus était constitué de 12 descriptions de la ville de Rouen (6 romans policiers et 4 romans, un récit de voyage et une autobiographie) et de 2 nouvelles policières au choix ayant pour cadre le même environnement. Il était présenté sous la forme d'un fascicule reproduisant l'ensemble des textes, ainsi que le paratexte les accompagnant ${ }^{(7)}$. La lecture des descriptions a été précédée d'un travail d'observation sur le paratexte à partir d'un ensemble diversifié de romans présents dans la classe et des ouvrages présentés dans le fascicule. Le projet a ensuite donné lieu à différentes activités, telle que l'observation des caractéristiques génériques des extraits descriptifs suivie par l'interprétation de ces observations. L'objectif était de pouvoir reproduire ces observations et ces analyses de manière autonome, lors de la lecture d'une nouvelle, puis lors de l'écriture de son propre texte.

Cette expérimentation a été suivie par des entretiens auprès des apprenants et de l'enseignant afin de mesurer la conscientisation de leur apprentissage et les acquis réalisés pendant le projet.

Le premier résultat porte sur le nombre de livres lus de manière autonome avant et après la réalisation du projet. Sur un groupe de 12 apprenants, seule une étudiante avait lu un livre en français. A la fin du semestre, soit quatre mois après la fin du projet, tous les étudiants ont lu au moins 2 livres, empruntés parfois à la bibliothèque, mais le plus souvent achetés à la librairie proche de l'université où une visite avait eu lieu avec l'enseignant. Interrogés sur les raisons de cette augmentation, ces nouveaux lecteurs en français ont répondu qu'ils avaient "gagné en assurance », qu'il leur était « plus facile de savoir choisir un livre » et qu'ils savaient désormais « comment lire ». Leur ayant fait remarquer qu'ils savaient déjà comment lire un roman, ils nous ont répondu qu'en français « avant, c'était pas pareil ».

La production en groupe d'une nouvelle policière de quelques pages leur a permis d'intérioriser le modèle prototypique de la nouvelle policière composée d'un crime et d'une enquête, et de s'approprier ce modèle, notamment en discutant avec leur binôme des choix à faire pour le respecter. Conscients des possibilités de transgression, ils s'y sont parfois essayés sur des points très précis, comme le détournement du dénouement ou l'utilisation d'un faux crime, en ayant chaque fois l'envie de surprendre le lecteur. Le bilan a donc été très positif pour les apprenants comme pour l'enseignant qui a observé une motivation accrue dès qu'il consacrait un temps du cours à ce projet.

Dans le cadre de ce projet, la lecture de plusieurs textes présentant un même type de séquence et portant sur un sujet identique a permis à un groupe d'apprenants de prendre conscience des variations génériques possibles, du jeu que l'auteur peut introduire dans son texte à destination du lecteur. Le choix d'un genre

(7) Comme le rappelle K. Canvat : « le cadrage générique constitue une opération essentielle pour l'établissement du contrat de lecture. Par cadrage, on entend ici l'activité sémiotique qui consiste à inférer un certain nombre d'informations à partir d'indices disposés à la périphérie du texte (dans le paratexte et le métatexte), à sa lisière (dans l'espace textuel et l'incipit), mais aussi dans le texte lui-même (les superstructures) » (1999:115). L'identification de l'appartenance générique d'un texte se fait tout autant avant que pendant la lecture. 
dont les codes sont connus comme peut l'être le genre policier a permis aux apprenants de s'appuyer sur leurs connaissances pour observer les textes et accéder à des textes plus complexes.

Le recours à l'écriture créative en classe a permis de valider les apprentissages et de mesurer l'intégration des savoir-faire visés. L'augmentation des lectures autonomes s'est également révélée être un bon indicateur de l'évolution des apprenants vers une posture de lecteur autonome, et de l'accès au livre et à un mode de lecture plus fluide et plus proche de la lecture en langue maternelle.

\section{Conclusion}

Si le genre peut paraitre à première vue sclérosé et inadapté pour décrire les œuvres littéraires actuelles, l'observation des pratiques de production et de réception montre qu'il reste un outil d'analyse indispensable. Complété d'outils théoriques comme les régimes de généricité qui permettent de décrire la participation générique d'un texte, d'un discours éditorial et de la réception par le lecteur, il permet de disposer d'un modèle relativement simple d'utilisation pour l'enseignant.

Dans la classe de langue étrangère, il ne s'agira toutefois pas d'apprendre les genres pour ce qu'ils sont mais de disposer d'une compétence générique contribuant à la compétence lectoriale et offrant à l'apprenant des savoirs et des savoir-faire lui permettant d'accéder à une posture de lecteur. Autonome, l'apprenant pourra ainsi disposer des outils nécessaires à la poursuite de son apprentissage dans et hors de la classe et à la pratique personnelle de la langue apprise.

L'accès au texte littéraire n'est pas indispensable à l'apprentissage d'une langue étrangère, mais la lecture littéraire vue comme un jeu, comme un espace de rencontre entre le lecteur et le texte, pourra mettre en confiance l'apprenant en lui offrant un accès direct à un élément prégnant de la culture cible.

La compétence générique apparait alors comme un préalable indispensable, comme une porte d'accès au texte et au plaisir de lire. 


\section{Bibliographie}

ADAM, J.-M. \& HEIDMANN, U. (2007) : « Six propositions pour l'étude de la généricité », in Baroni R., Macé M., Le savoir des genres, Presses universitaires de Rennes, La licorne ${ }^{\circ} 79$, p. 21-34.

ANSEL, Y. (2005) : " Sociologie des marges littéraires », in Forest, P., Szkilnik, M., Théorie des marges littéraires, Université de Nantes, éd. Cécile Defaut, p. 73-130.

BOURDIEU, P. (1992) : Les règles de l'art, genèse et structure du champ littéraire, Éditions du Seuil.

CANVAT, K. (1999) : Enseigner la littérature par les genres. Pour une approche théorique et didactique de la notion de genre littéraire, De Boeck Duculot.

COMBE, D. (1992) : Les genres littéraires, Hachette.

Compagnon, A. (2007 [réf. du 31 septembre 2007]) : Théorie de la littérature : la notion de genre, [en ligne], disponible sur : http ://www.fabula.org/compagnon/genre.php

DUFAYS, J.-L. (1994) : Stéréotype et lecture, Mardaga.

Dufays, J.-L., GEMENNE, L., LEDUR, D. (2005) : Pour une lecture littéraire : histoire, théories, pistes pour la classe, De Boeck.

ECO, U. (1985) : Lector in fabula, le rôle du lecteur ou la coopération interprétative dans les textes narratifs, Le livre de poche.

HeRSENT, J.-F. (2000) : Sociologie de la lecture en France : état des lieux, Direction du livre et de la lecture, Ministère de la culture.

LANE, P. (1992) : La périphérie du texte, Nathan université.

RIQUOIS, E. (2009) : «Le texte littéraire dans les manuels de français langue étrangère : un document authentique parmi d'autres ? », in Dufays J.-L. (dir), Le langage et l'Homme, «Le retour du littéraire en classe de langue-culture », vol. XXXXIV, $n^{\circ} 1$, E.M.E., p. 3-16.

- (2010a) : «Acquérir une compétence lectoriale en français langue étrangère et seconde ", in Neveu, F., MuniToke, V. (et alii), Actes du Congrès Mondial de Linguistique Française, Institut de Linguistique Française.

— (2010b) : «La linguistique textuelle au service des genres littéraires », in Ablali, D., Kastberg, M. (éds), Actes du colloque linguistique et littérature : Cluny, 40 ans après, Laseldi, Université de Franche-Comté, Centre de recherches Jacques-Petit. En ligne: http ://alufc.univfcomte.fr/pdfs/913/pdf_18.pdf

SCHAEFFER, J.-M. (1989) : Qu'est-ce qu'un genre littéraire?, Éditions du Seuil, coll. Poétique.

SÉoud, A. (1997) : Pour une didactique de la littérature, Crédif, Hatier/Didier.

TomacheVsKi, B. V. (1965 [réédition de 2001]) : «Thématique », in Todorov, T. (dir.), Théories de la littérature, Textes des formalistes russes, Éditions du Seuil, coll. Points essais, p. 267-312. 